SHORT REPORT

\title{
Systemic infection, interleukin $1 \beta$, and cognitive decline in Alzheimer's disease
}

\author{
C Holmes, M El-Okl, A L Williams, C Cunningham, D Wilcockson, V H Perry
}

J Neurol Neurosurg Psychiatry 2003;74:788-789

Activated microglia, the resident macrophages of the brain, are a feature of Alzheimer's disease. Animal models suggest that when activated microglia are further activated by a subsequent systemic infection this results in significantly raised levels of interleukin $1 \beta$ within the CNS, which may in turn potentiate neurodegeneration. This prospective pilot study in Alzheimer's disease subjects showed that cognitive function can be impaired for at least two months after the resolution of a systemic infection and that cognitive impairment is preceded by raised serum levels of interleukin $1 \beta$. These relations were not confounded by the presence of any subsequent systemic infection or by baseline cognitive scores. Further research is needed to determine whether recurrent systemic infections drive cognitive decline in Alzheimer's disease subjects through a cytokine mediated pathway.

t is well recognised that following a systemic infection patients with Alzheimer's disease are susceptible to developIng delirium, in which their premorbid cognitive state is further impaired. This acute on chronic cognitive impairment is usually short lived (less than two weeks) but in some cases may be prolonged beyond the episode of acute delirium. ${ }^{1}$ In older patients delirium is an independent predictor of sustained poor cognition at one year follow $u^{2}$ and of dementing illness at two years. ${ }^{3}$ Whether systemic infection can cause prolonged cognitive impairment in patients with Alzheimer's disease when delirium is absent is not known. The reason why systemic infection, in the absence (or presence) of a delirium, could cause additional prolonged cognitive impairment in Alzheimer's disease is also unknown.

The systemic response to infection is accompanied by increased circulating levels of cytokines such as interleukin 6 and interleukin $1 \beta$ (IL- $1 \beta)$, though the latter is typically measurable only in more severe infections. ${ }^{4}$ We have shown in an animal model of chronic neurodegeneration in which brain microglia are activated that a peripheral challenge with endotoxin, intended to mimic a peripheral infection, is associated with increased secretion of IL- $1 \beta$ within the central nervous system. ${ }^{5}$ We hypothesised that in Alzheimer's disease a systemic infection will act as a potent secondary stimulus to the brain microglia because these microglia in the Alzheimer's disease brain are already "primed" by the presence of $\beta$ amyloid and degenerating neurones. Thus the levels of cytokines in the brain will become raised, initiating a positive feedback cycle that gives rise to an increasing accumulation of pathological changes and cognitive decline that is characteristic of Alzheimer's disease. ${ }^{6}$

We anticipated that, even in the absence of delirium, patients with Alzheimer's disease would show clinical evidence of permanent cognitive decline following a systemic infection. In addition, we hypothesised that the presence of raised serum concentrations of IL- $1 \beta$ would be associated with this permanent cognitive decline.

\section{METHODS}

Ninety two community dwelling patients, fulfilling NINCDSADRDA diagnostic criteria for Alzheimer's disease, took part in the study. Of these, seven had evidence on physical examination of a current systemic infection at baseline and were excluded from the study.

The remaining 85 patients, with no evidence of systemic infection at baseline, were interviewed using the mini-mental state examination (MMSE), were assessed for the presence of delirium using the confusion assessment method (CAM), ${ }^{7}$ and a blood sample was taken. Blood samples were analysed for IL-1 $\beta$ using a human IL-1 $\beta$ sandwich enzyme linked immunosorbent assay (ELISA; R\&D Systems, Abingdon, UK), which was carried out with undiluted sera according to the manufacturer's instructions. A detailed systematic history was then taken from the carers of these patients regarding the presence or absence of systemic infections in the two months before the baseline examination of the patient.

The patient and main carer were revisited two months later. The patient was re-examined, looking for evidence of a current systemic infection, and the CAM and MMSE were repeated. The main carer was reinterviewed regarding the presence or absence of any systemic infection in the two month period between the baseline examination of the patient and the follow up examination.

The relation between cognitive decline and both the presence of systemic infection in the two month period before the baseline examination and the presence of detectable IL-1 $\beta$ levels at baseline was examined using an unpaired $t$ test. Possible confounding factors for subsequent systemic infection and the baseline cognitive score were examined using linear regression analysis.

\section{RESULTS}

The mean (SD) age of the 85 patients followed up in the study was 86.5 (7.8) years; $66(77 \%)$ of them were female. The patients had a mean MMSE score of 15.9 (6.8) points at baseline, with a rate of cognitive change of -0.1 (3.3) points over the two month study period. No patients fulfilled CAM diagnostic criteria for delirium at baseline or at follow up examination.

Fifteen patients $(16 \%)$ had evidence of a systemic infection in the two months preceding the baseline examination. These patients showed a greater rate of cognitive decline over the two month follow up period when compared with those who were free of infection in the two months preceding baseline examination (MMSE decline - 1.6 (3.4) $v+0.2$ (3.1) points; one tailed $t$ test, $\mathrm{p}=0.02$ )

Sixty five $(76 \%)$ of the 85 patients and their carers gave permission for blood sampling for cytokine levels at their baseline examination. Six patients $(9 \%)$ had detectable blood serum levels of IL- $1 \beta$ (defined as $>2.5 \mathrm{pg} / \mathrm{ml}$ ). These patients had an 
increased rate of cognitive decline over the two month study period compared with those with no detectable levels of IL- $1 \beta$ (MMSE decline -2.2 (3.6) $v+0.4$ (3.4) points; one tailed $t$ test, $\mathrm{p}=0.03)$.

Among the 15 patients who had evidence of a systemic infection in the two months preceding baseline examination, there were only three carers who gave permission for blood sampling for cytokine levels and none of these subjects had detectable blood serum levels of IL- $1 \beta$.

Of the 85 patients in the study, 16 (19\%) developed a systemic infection during the two month follow up period. The presence of a systemic infection during the follow up period was associated with an increased rate of cognitive decline, although this did not reach significance (MMSE decline -1.2 (2.6) $v+0.1$ (3.3) points; one tailed $t$ test, $\mathrm{p}=0.08)$. Of these 16 patients, five $(31 \%)$ had a previous infection before baseline and $11(69 \%)$ had no previous infection before baseline. The presence of a systemic infection during the follow up period was not significantly associated with an increased frequency of infection before baseline examination (33\% (5/15) v $14 \%$ ( $11 / 70)$; Fisher's exact test, $p=0.14)$. Linear regression analysis showed that the relation between systemic infection in the two months preceding baseline examination and the raised serum IL- $1 \beta$ levels associated with an increased rate of cognitive decline was not confounded by the development of a systemic infection over the two month follow up period.

There was no significant difference in the MMSE score at baseline examination in those patients who had evidence of a systemic infection before baseline compared with those who did not ( $18.2(6.2) v 15.6(6.9)$ points; $t$ test, $\mathrm{p}=0.2)$. However, there was evidence of a correlation between high MMSE scores at baseline and an increased rate of cognitive declinethat is, patients with a high MMSE score at baseline declined more quickly (Pearson correlation $-0.22, \mathrm{p}=0.04$ ). Linear regression analysis showed that the relation between systemic infection in the two months preceding the baseline examination and the raised serum IL-1 $\beta$ levels associated with an increased rate of cognitive decline was not confounded by baseline MMSE scores.

\section{DISCUSSION}

While every attempt was made to record every episode of infection, it is recognised that the retrospective diagnosis of systemic infection in elderly people can be problematic and some infections may have been missed. In addition, the small number of patients with a history of infection who also gave permission for cytokine assays did not enable us to confirm (or exclude) a relation between systemic infection and cytokine production.

However, this pilot study shows that both systemic infection and raised serum IL- $1 \beta$ levels are associated with a greater rate of cognitive decline in patients with Alzheimer's disease, and that the presence of a systemic infection can continue to impair cognitive performance two months after the resolution of the infection. These relations did not appear to be confounded by the presence of a subsequent infection or by (non-significant) differences in baseline cognitive scores. Furthermore, the cognitive decline found in this study occurred in the absence of any evidence of delirium.

A longer follow up period is clearly required to examine whether the cognitive deterioration following a systemic infection persists. However, this study raises the possibility that recurrent systemic infection (here present in between $16 \%$ and $19 \%$ of patients in any two month period) may help to drive cognitive decline in Alzheimer's disease through cytokine mediated pathways, and may also provide an explanation for the protective effects of non-steroidal anti-inflammatory $\operatorname{drugs}^{8}$ in affected patients.

\section{ACKNOWLEDGEMENTS}

We would like to acknowledge the support of the Medical Research Council and the Wellcome Trust for laboratory staffing costs.

\section{Authors' affiliations}

C Holmes, M El-Okl, A L Williams, C Cunningham, D Wilcockson,

V H Perry, School of Medicine and Biological Sciences, University of

Southampton, Southampton, UK

Competing interests: none declared

Correspondence to: Dr Clive Holmes, University of Southampton, Clinical Neurosciences Research Division, Memory Assessment and Research Centre, Moorgreen Hospital, Botley Rd, West End, Southampton SO30 3JB, UK; ch4@soton.ac.uk

Received 27 September 2002

In revised form 12 December 2002

Accepted 21 December 2002

\section{REFERENCES}

1 Manos PJ, Wu R. The duration of delirium in medical and post-operative patients referred for psychiatric consultation. Ann Clin Psychiatry 1997;9:219-26.

2 McCusker J, Cole M, Dendukuri N, et al. Delirium in older medical inpatients and subsequent cognitive and functional status: A prospective study. Can Med Assoc J 2001;165:575-83.

3 Rankonen T, Luukkainen MR, Paanila S, et al. Delirium episode as a sign of undetected dementia among community dwelling elderly subjects: a 2 year follow up study. I Neurol Neurosurg Psychiatry 2000;69:519-21.

4 Baver T, Monton C, Torres A, et al. Comparison of systemic cytokine levels in patients with acute respiratory distress syndrome severe pneumonia and controls. Thorax 2000;55:46-52.

5 Combrinck MI, Perry VH, Cunningham C. Peripheral infection evokes exaggerated sickness behaviour in pre-clinical murine prion disease. Neuroscience 2002:112: 7-11.

6 Griffin WS, Sheng JG, Royston SM, et al. Glial neuronal interactions in $A D$ : the potential role of a cytokine cycle in disease progression. Brain Pathology 1998;8:65-72

7 Inouye SK, van Dyck CH, Alessi CA, et al. Clarifying confusion: the confusion assessment method. Annals Intern Med 1990;113:941-8.

8 Veld BA, Ruitenberg A, Hofman A, et al. Nonsteroidal antiinflammatory drugs and the risk of Alzheimer's disease. N Engl J Med 2001;345: 1515-21. 